'Departamento de Radiología, Sección Medicina Nuclear, Clínica Santa María. Santiago, Chile. 2Departamento de Cirugía, Clinica Santa María. Santiago, Chile. 3Dirección Académica, Clínica Santa María, Santiago, Chile. aTecnólogo Médico.

Recibido el 28 de mayo de 2015 , aceptado 15 de octubre de 2015 .

Correspondencia a: Pamela Humeres Aprá Sección Medicina Nuclear Clínica Santa María Dirección: Av. Santa María 0410, 1 er piso Rayos, Sección Medicina Nuclear.

Teléfono: +56229133094 phumeres@clinicasantamaria.cl

\section{Linfonodo centinela en cáncer de mama: correlación entre detección isotópica y quirúrgica}

\author{
PAMELA HUMERES A. ${ }^{1}$, JAVIERA GONZÁLEZ ${ }^{1}$, PATRICIO GONZÁLEZ1, \\ ENRIQUE WAUGH ${ }^{2}$, FERNANDO GÓMEZ ${ }^{2}$, ISABEL ALLIENDE 3 ,a
}

\section{Pre and intraoperative scintigraphic detection of sentinel nodes in breast cancer}

Background: Sentinel node detection localizes the first node that drains a malignant lesion aiming to detect tumor dissemination. Aim: To assess the yield of sentinel node detection in breast cancer, using pre or intraoperative scintigraphy. Material and Methods: Review of medical records of patients with breast cancer who had a scintigraphic detection of sentinel nodes. Lymph node scintigraphy and surgery were performed in the same day. Results: We studied 174 women aged $53 \pm 13$ years, operated with a diagnosis of breast cancer, including six highly suspicious lesions in the contralateral breast (totaling 180 studied breasts). Preoperative scintigraphy showed a sentinel node in 174 of 180 breasts (97\%). Intraoperative gamma probe confirmed the presence of the sentinel node in the same 174 breasts and detected an additional one reaching a detection yield of $97 \%$. Four patients in whom a sentinel node was not detected in the preoperative scintigraphy, had macrometastases. Frozen section biopsies were available in 177 of 180 breasts. Metastases were informed in 45 patients who underwent axillary lymph node dissection, plus one additional patient with a suspicious lesion. Conclusions: A high rate of sentinel node detection in the preoperative scintigraphy was observed. Most sentinel nodes not detected with nuclear medicine had macrometastases. In $71 \%$ of patients, the detection of sentinel node avoided axillary lymph node dissection.

(Rev Med Chile 2016; 144: 66-73)

Key words: Breast neoplasms; Lymphatic metastasis; Radionuclide imaging; Sentinel lymph node biopsy.

\section{L}

a técnica de detección del linfonodo centinela (LC) fue descrita por Morton, en el año 1992, en pacientes con melanoma, usando azul de isosulfán ${ }^{1}$. El objetivo es localizar el o los primeros linfonodos que drenan un territorio con una lesión maligna, para evaluar el compromiso microscópico de éste, lo que años antes había sido propuesto por Cabañas'. En 1993, Alex y Krag demostraron, en un modelo animal, que tanto el azul de isosulfán, como los radiotrazadores eran igualmente efectivos en localizar el LC; posteriormente varios otros estudios lo confirmaron ${ }^{3-6}$.
En el estudio de las lesiones malignas de mama, con axila clínicamente negativa, el estado histológico de los linfonodos axilares es reconocidamente uno de los factores más importantes para el pronóstico y tratamiento de la enfermedad.

La disección de linfonodo axilar (DLA) ha sido rutinariamente usada en el tratamiento y estadificación del cáncer de mama en estadio inicial, con beneficios en el control de la enfermedad y pronóstico, pero la alteración anatómica de la DLA puede resultar en linfedema, disfunción del hombro, daño en la inervación, con el consiguien- 
te deterioro de la calidad de vida ${ }^{7-13}$. En pacientes con axila clínicamente positiva o con compromiso metastásico confirmado por otros métodos diagnósticos, como punción con aguja fina bajo ecografía, la DLA permanece como técnica estándar, pero en pacientes con axila clínicamente negativa, el estudio del LC es una forma de etapificar con menos morbilidad que la disección axilar y así evitar cirugías extensas ${ }^{1,14,15}$.

Uno de los primeros estudios multicéntricos en cáncer de mama con detección de LC con radioisótopos y sonda o gamma intraoperatorio fue publicado por $\mathrm{Krag}^{16}$ y evaluó a 11 distintos cirujanos. De 443 pacientes con cáncer de mama usando radiocoloide marcado con Tecnecio $99 \mathrm{~m}$ (Tc99m)y posterior DLA, se encontró una tasa de detección de $93 \%$. El compromiso anatomopatológico del LC se comparó con los restantes linfonodos axilares. La certeza diagnóstica fue de $97 \%$, la especificidad de $100 \%$, la sensibilidad de $89 \%$, el valor predictivo positivo de $100 \%$ y el negativo fue $96 \%$. Concluyeron que la biopsia de LC puede predecir la presencia o ausencia de compromiso metastásico axilar en cáncer de mama, aunque la tasa de éxito, varía según el cirujano.

En la literatura,el porcentaje de detección del LC es variable, pero en promedio es de alrededor de $96 \%{ }^{14,17} \mathrm{y}$, según la gran mayoría de series, predice el compromiso del resto de los linfonodos axilares en más de 95\% de los casos, observándose aumento en su utilización. En Estados Unidos de Norteamérica, la base de datos del National Cancer Database mostró incremento en el uso la técnica del LC de $27 \%$ a $66 \%$, entre 1998 y 2005. De 490.000 pacientes con cáncer mamario inicial, se evidenció que la mayoría de los centros de cáncer y cirujanos están realizando la detección del LC. Datos similares publicaron estudios de Canadá y Reino Unido ${ }^{18-20}$.

Las guías de la Sociedad Americana de Cirujanos de Mama (SACM) recomiendan que cada cirujano documente su experiencia y que alcance sobre $85 \%$ de detección, con falsos negativos menor o igual a $5 \%$ antes de dejar la DLA. No está claramente establecido el número de casos que debe realizar un cirujano para realizar el procedimiento con seguridad, pero la SACM y la American Society of Clinical Oncology (ASCO) recomiendan un mínimo de 20 casos de LC con sus respectivas DLA, para disminuir al mínimo la posibilidad de falsos negativos ${ }^{15}$.
Las indicaciones más ampliamente aceptadas en el uso del LC son:

1) Mujeres con axila clínicamente negativa, con cáncer de mama microinvasor o invasor ${ }^{7-15}$.

2) Mujeres con carcinomas in situ extensos quienes serán sometidas a mastectomía total.

En nuestro estudio hemos querido realizar una revisión retrospectiva del rendimiento del LC usando radiocoloide y su posterior correlación con la detección quirúrgica mediante gamma probe intraoperatorio, en pacientes con cáncer de mama, en quienes el cirujano solicitó el estudio de LC, realizados en Clínica Santa María entre marzo de 2006 y agosto de 2011.

\section{Materiales y Método}

Se realizó una revisión retrospectiva de los datos disponibles en el registro clínico electrónico (RCE) y base de datos de radiología de Clínica Santa María, de todos los pacientes ingresados entre marzo de 2006 y agosto de 2011 para cirugía por cáncer de mama, en que se solicitó el estudio prequirúrgico de LC con técnica isotópica. Se obtuvo la información completa en 174 pacientes. Se incluyeron todos los pacientes que contaban con biopsia mamaria compatible con lesión primaria maligna. No se discriminó por tamaño tumoral, ni tipo histológico. Para el análisis se consideraron 180 mamas, 6 casos por lesión sospechosa contralateral a las que también se realizó LC. Se cuenta con la aprobación del Comité de Ética de Clínica Santa María.

La técnica de LC consistió en 2 inyecciones retroareolares de 0.1 a $0.2 \mathrm{ml}$, con 100 a $200 \mathrm{mi}$ crocuries de Tc99m nanocoloides, (Nanocint de laboratorio CGM Nuclear, Chile). Se realizó masaje local sobre la inyección por $5 \mathrm{~min}$, para acelerar la migración del radiofármaco y la visualización del LC. Inmediatamente después de la inyección, se adquirieron imágenes dinámicas en gamma cámara Genesys Dual Head, Adac, por 30 min. De no visualizarse el LC en este período, se realizaron controles tardíos a la hora y, si fuera necesario, a las 2 y $3 \mathrm{~h}$. Se adquirieron proyecciones anterior y lateral (Figuras 1 y 2). Para fines de localización los LC intramamarios son considerados como de axila correspondiente.

En pabellón, la detección del LC se realizó 
con sonda de radiación gamma Navigator, Dilon. Para el análisis de este trabajo, no se consideró la inyección del azul de isosulfán, pues no todos los protocolos quirúrgicos tenían la descripción de esta técnica (descrita en 71,6\%). Una vez abierta la fascia axilar, el cirujano identificó el o los LC, disecando aquellos con cuentas elevadas. El cri-

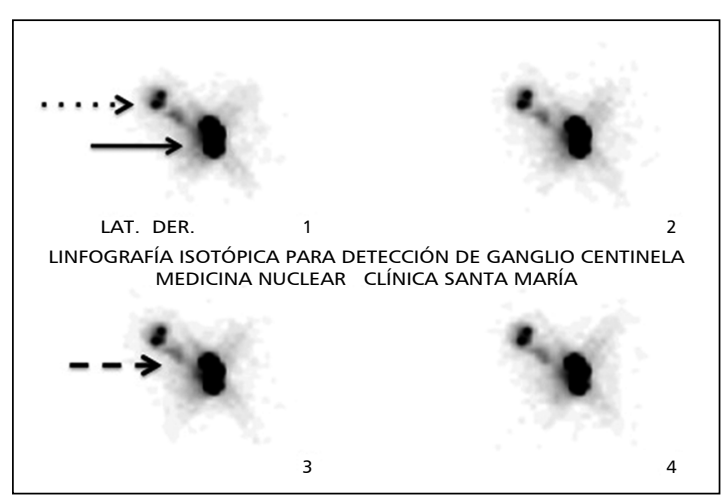

Figura 1. Paciente con linfocintigrafía con Tc99m-Nanocint en mama derecha. Flecha punteada: 2 LC. Flecha lisa: sitio de inyección. Flecha de guiones: trayecto linfático. terio para positividad con gamma probe incluyó a todos aquellos LC que tenían más de $10 \%$ de emisión respecto del más captante.

El o los LC resecados fueron enviados a biopsia rápida, donde se le realizó impronta. Si se informaba a lo menos un LC con metástasis, se procedió a DLA. Si los LC fueron negativos, no se realizó DLA.

El estudio cintigráfico se realizó por dos médicos entrenados en medicina nuclear y, en pabellón, el uso del gamma probe fue realizado en la mayoría de los pacientes,por dos cirujanos previamente entrenados en la técnica.

Para la estadística, se realizó un análisis descriptivo de las variables de estudio. Para variables cuantitativas se calcularon promedios y desviaciones estándar y para variables cualitativas se estudió la distribución de frecuencias. Se calcularon intervalos de confianza para la proporción de detección de LC por medicina nuclear, y se calculó el coeficiente kappa con su respectivo intervalo de confianza para estudiar la concordancia entre los resultados de las biopsias rápida y definitiva. Para estudiar la relación entre variables cualitativas se utilizó el test de $\chi^{2}$.

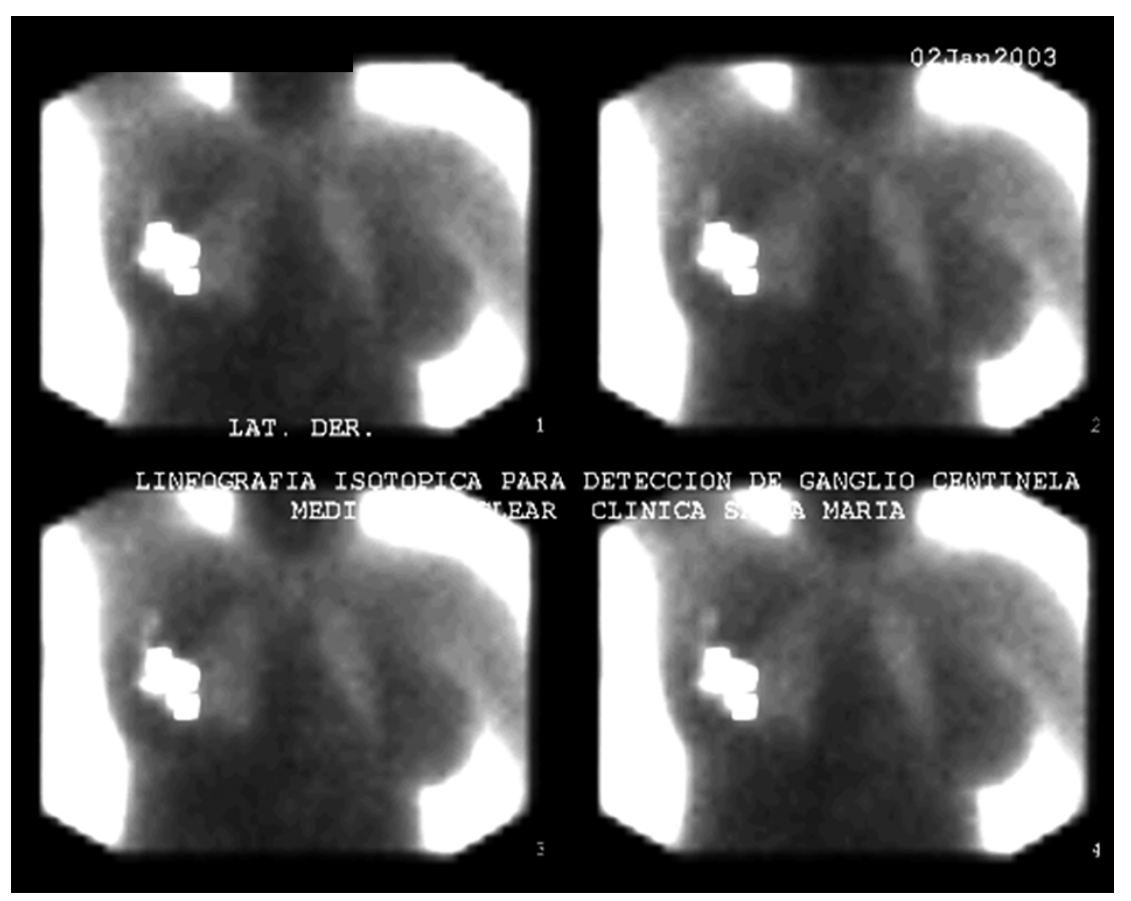

Figura 2. Paciente de Figura 1 con fantoma de Tecnecio, lo cual permite ver contornos del cuerpo. 
Los análisis se realizaron con el software STATA 13 y se consideró una confianza de 95\%.

\section{Resultados}

Del RCE y base de datos de radiología, se obtuvieron 245 pacientes con estudio de LC preoperatorio en cáncer de mama, pero se contó con los resultados cintigráficos y hallazgos operatorios en 174 pacientes, que fueron los incluidos en este estudio.

Todos los pacientes tenían biopsia core de la lesión mamaria compatible con malignidad. Los datos clínicos de los pacientes y ubicación de las lesiones mamarias están en la Tabla 1. En total, se analizaron 180 mamas, ya que 6 pacientes tenían estudio bilateral por lesión contralateral sospechosa.

En 174 mamas se realizó la detección preoperatoria del $\mathrm{LC}$, que corresponde a una tasa de detección de $96,7 \%$, con un intervalo de confianza (IC) de 92,9-98,8\%. El 100\% correspondió a la axila del mismo lado de la lesión mamaria. Hubo $4 \%$ con visualización simultánea de LC en cadena mamaria interna.

En pabellón, los 174 LC previamente visualizados en la linfocintigrafía se detectaron con gamma probe. Sólo hubo un paciente no detectado previo a la cirugía y si con gamma probe intraoperatorio, aunque con pocas cuentas, sin metástasis en la biopsia rápida, que fue a DLA. Esto aumenta de tasa de detección del LC a 97,2\% (IC de 93,6-99,1). Cuatro de los 5 pacientes sin visualización previa del LC, tenían macrometástasis (80\%).

Contamos con la biopsia rápida en 177 de 180 mamas. En estas se informó compromiso metastásico en $45(25,4 \%)$, sin metástasis en $131(74 \%)$ y una dudosa $(0,6 \%)$. En la cirugía, se realizaron 46 DLA, correspondientes a los 45 LC con metástasis en la biopsia rápida y al LC con pocas cuentas (dudoso).

Los 180 pacientes tenían biopsias definitivas. En 9 de ellos hubo discordancia entre la biopsia rápida y la definitiva, todos con micrometástasis en la definitiva. En 7/9 se completó DLA en un segundo tiempo. En los otros 2 pacientes también con micrometástasis, no se realizó la DLA, ya que el estudio de diseminación mostró lesiones secundarias. Para el total de mamas estudiadas hubo alta concordancia entre biopsias rápidas y definitivas con un coeficiente kappa de 0,862 (Tabla 2).
Tabla 1. Características clínicas de los pacientes y ubicación de la lesión mamaria (expresado como porcentaje o promedio \pm DE)

\begin{tabular}{|c|c|c|c|}
\hline \multicolumn{4}{|c|}{ Descripción pacientes estudiados $(n=174)$} \\
\hline \multicolumn{2}{|c|}{ Variable } & \multicolumn{2}{|c|}{ Promedio \pm DE / \% (n) } \\
\hline Edad & & \multicolumn{2}{|c|}{$52,7 \pm 13,1$} \\
\hline Sexo & $\begin{array}{l}\text { Mujeres } \\
\text { Hombres }\end{array}$ & $\begin{array}{r}98,9 \% \\
1,1 \%\end{array}$ & $\begin{array}{r}(172) \\
(2)\end{array}$ \\
\hline Mama & $\begin{array}{l}\text { Derecha } \\
\text { Izquierda } \\
\text { Ambas }\end{array}$ & $\begin{array}{r}50,0 \% \\
46,6 \% \\
3,4 \%\end{array}$ & $\begin{array}{r}(87) \\
(81) \\
(6)\end{array}$ \\
\hline
\end{tabular}

\section{Tabla 2. Resultado entre biopsias rápida y} definitiva en lesiones mamarias

\begin{tabular}{|lrr|}
\hline Biopsia rápida & & \\
Positiva & $25,4 \%$ & $(45 / 180)$ \\
Negativa & $72,8 \%$ & $(131 / 180)$ \\
Sospechosa & $0,6 \%$ & $(1 / 180)$ \\
No realizada & $1,7 \%$ & $(3 / 180)$ \\
Biopsia definitiva & & \\
Positiva & $100,0 \%$ & $(45 / 45)$ \\
Positiva & $6,9 \%$ & $(9 / 131)$ \\
Negativa & $93,1 \%$ & $(122 / 131)$ \\
Negativa & $100,0 \%$ & $(1 / 1)$ \\
Negativa & $100,0 \%$ & $(3 / 3)$ \\
\hline
\end{tabular}

Se calculó el coeficiente Kappa para evaluar la concordancia entre los resultados de las biopsias rápidas y definitivas. Se obtuvo un coeficiente Kappa de 0,862 con un intervalo de confianza de $95 \%$ de $(0,835 ; 0,903)$, resultado que indica una concordancia alta entre ambos estudios.

\section{Discusión}

La técnica del LC está basada en la observación que las células tumorales migran hacia uno o algunos linfonodos, llamados centinelas, antes de comprometer las siguientes estaciones linfonodales. La inyección de coloide marcado con Tc99m permite localizar el o los LC en la mayoría de los pacientes y su estado histológico predeciría el compromiso metastásico de los restantes linfonodos en más de $95 \%$ de los casos $^{1-3}$. Generalmente, el LC que recibe el drenaje linfático mamario está ubicado en el primer nivel de los linfonodos axilares, pero han sido descritas otras localizaciones, como la axila contralateral en pacientes con disección previa ${ }^{4}$. 
En el cáncer mamario inicial, el estado histológico de los linfonodos es uno de los factores más importantes en el pronóstico. La DLA ha sido usada de rutina en el tratamiento de un cáncer mamario inicial para mejorar su pronóstico, pero puede producir efectos colaterales como linfedema y otras alteraciones que comprometen la calidad de vida ${ }^{7-13}$. Se ha descrito $2 \%$ de linfedema en pacientes con centinela, comparado con $13 \%$ en pacientes con DLA?

Nuestra tasa de detección de LC preoperatoria fue 96,7 y $97,2 \%$, si consideramos los detectados en la cirugía y este resultado es similar a lo publicada por la literatura ${ }^{14,17}$. Estos porcentajes, probablemente son explicados porque ambas técnicas fueron realizadas por 2 cirujanos y médicos nucleares con experiencia. Según la guía clínica de la ASCO, basada en 69 trabajos, en 8.059 pacientes, se identifica el LC usando coloide y/o azul de isosulfán en $95 \%$ de los pacientes ${ }^{15}$. La combinación de ambos métodos tiene mejor tasa de detección del $\mathrm{LC}^{15,21}$.

Nuestro porcentaje de DLA $(29,4 \%)$ es algo mayor al publicado en la literatura y probablemente explicable porque no hubo exclusión de tumores por histología ni tamaño. Se ha descrito que el compromiso axilar considerando el grado histológico es de $3 \%$ para tumores de bajo grado y $21 \%$ para los de grado 2 y $3^{22}$.

La mayoría de los LC no visualizados prequirúrgicamente presentaron macrometástasis $(80 \%)$. Varios trabajos han descrito que en caso de metástasis linfonodal masiva, el flujo del coloide es derivado a otro linfonodo. Esto refuerza el concepto que no sólo debe extirparse el más "caliente" o radiactivo, sino también cualquier linfonodo palpable sospechoso ${ }^{23,24}$. En el estudio NSABP B-32, la remoción de 2 LC disminuye a la mitad la tasa de falsos negativos ${ }^{25}$.

En los pacientes que tengan LC positivo, se ha publicado que $40 \%$ de ellos tendrán enfermedad axilar remanente $e^{3,4,15,21,26-32}$. Por enfermedad remanente se considera tanto micro como macrometástasis. Existe controversia respecto al manejo de las submicrometástasis (compromiso $<$ a 0,2 mm) o células tumorales aisladas, las que se consideran en general como linfonodos negativos para efectos de pronóstico. En el caso de LC positivo, tanto para las macrometástasis (compromiso $>2 \mathrm{~mm}$ ) como micrometástasis, las guías de la ASCO 2014 y NCCN (National Comprehensive Cancer Network) recomiendan no realizar la DLA, para pacientes que tengan hasta 2 LC positivos, y que sean tratados con cirugía conservadora más radioterapia ${ }^{33}$.

Aproximadamente la mitad de los casos con LC positivos en la cirugía tienen enfermedad residual axilar y se debe plantear ir a DLA. La mayoría de los cirujanos realiza la disección en el mismo tiempo quirúrgico cuando la biopsia rápida demuestra metástasis en el LC, aunque algunos, para no añadir tiempo a la cirugía, prefieren realizarla en forma diferida o para discutir en comité o con su paciente los riesgos y beneficios de realizar o no una DLA. En nuestro estudio, con la biopsia rápida positiva, se realizó la DLA en el mismo tiempo quirúrgico, aunque no hay clara evidencia de la ventaja de una sobre la otra ${ }^{34}$.

Encontramos una alta correlación entre las biopsias rápidas y las definitivas. Las discordancias fueron en 9 casos por micrometástasis en las definitivas. A criterio del cirujano, 7 fueron a DLA en segundo tiempo. En 2 pacientes con compromiso secundario en el estudio de diseminación, no se realizó DLA. Los pacientes con compromiso micrometástasico tienen peor pronóstico que los con LC negativo, aunque la DLA es controversial realizarla en pacientes con menos de $3 \mathrm{LC}$, que recibirán radioterapia y con receptores de estrógenos positivos $33,35,36$.

En caso de LC extra-axilares, los intramamarios fueron catalogados como axilares y en el caso de los que drenaron a la cadena mamaria interna, no se hizo resección, a pesar que algunos de los pacientes presentaron contraste de éstos. Algunos trabajos han mostrado que linfonodos de la cadena mamaria interna, con axila negativa, pueden tener compromiso metastásico, pero su disección es controversial y permanece en investigación ${ }^{37-42}$. El rol del compromiso metastásico de estos LC no está claro, ya que en general, los seguimientos son para los LC axilares ${ }^{43,44}$.

Respecto a la técnica isotópica, nosotros realizamos punción retroareolar con nanocoloides, que son los más ampliamente usados, pero se usan otros radiocoloides, según disponibilidad. Se recomiendan los radiofármacos de tamaño pequeño. El sitio de la inyección puede ser perilesional, subdérmico, sobre el tumor o periareolar. Aparentemente habría mejor posibilidad de visualización del LC con la punción retroareolar y subareolar, que fue la usada en este estudio ${ }^{45}$.

Hay controversia sobre de la adquisición de 
imágenes prequirúrgicas, ya que en la mayoría de los casos el drenaje será a la axila correspondien$\mathrm{te}^{46}$. En nuestro estudio, de rutina se adquieren imágenes en todos los pacientes.

En nuestra revisión hemos podido comprobar la alta detección de LC con técnica isotópica, tanto antes de la cirugía, como en pabellón al ser realizada por cirujanos y médicos nucleares entrenados. Nuestros resultados de compromiso axilar y subsecuentemente de DLA, también son similares a lo publicado en la literatura. El uso de la técnica de LC evitó DLA innecesarias en aproximadamente dos tercios de pacientes con LC negativo.

En nuestra experiencia la técnica de LC es un método útil y sencillo de realizar en la estadificación axilar de los cánceres de mama en etapas precoces.

\section{Referencias}

1. Morton DL, Wen DR, Wong JH, Economou JS, Cagle LA, Storm FK et al. Technical details of intraoperative lymphatic mapping for early stage melanoma. Arch Surg 1992; 127 (4): 392-9.

2. Cabañas RM. An approach for the treatment of penile carcinoma. Cancer 1977; 39 (2): 456-66.

3. Krag DN, Weaver DL, Alex JC, Fairbank JT. Surgical resection and radiolocalization of the sentinel lymph node in breast cancer using a gamma probe. Surg Oncol 1993; 2 (6): 335-9.

4. Krag DN, Anderson SJ, Julian TB, Brown AM, , Ashikaga $\mathrm{T}$, et al. Technical outcomes of sentinel-lymph-node resection and conventional axillary-lymph-node dissection in patients with clinically node-negative breast cancer: results from the NSABP B-32 randomised phase III trial. Lancet Oncol 2007; 8 (10): 881-8.

5. Albertini JJ, Lyman GH, Cox C, Yeatman T, Balducci $\mathrm{L}, \mathrm{Ku} \mathrm{N}$, et al. Lymphatic mapping and sentinel node biopsy in the patient with breast cancer. JAMA 1996; 276 (22): 1818-22.

6. Cody HS 3rd, Fey J, Akhurst T, Fazzari M, Mazumdar $\mathrm{M}$, Yeung $\mathrm{H}$, et al. Complementarity of blue dye and isotope in sentinel node localization for breast cancer: univariate and multivariate analysis of 966 procedures. Ann Surg Oncol 2001; 8 (1): 13-9.

7. Lucci A, McCall LM, Beitsch PD, Whitworth PW, Reintgen DS, Blumencranz PW, et al. Surgical complications associated with sentinel lymph node dissection (SLND) plus axillary lymph node dissection compared with SLND alone in the American College of Surgeons
Oncology Group Trial Z0011. J Clin Oncol 2007; 25 (24): 3657-63.

8. Mansel RE, Fallowfield L, Kissin M, Goyal A, Newcombe RG, Dixon JM, et al. Randomized multicenter trial of sentinel node biopsy versus standard axillary treatment in operable breast cancer: the ALMANAC Trial. J Natl Cancer Inst 2006; 98 (9): 599-609.

9. Fleissig A, Fallowfield LJ, Langridge CI, Johnson L, Newcombe RG, Dixon JM, et al. Post-operative arm morbidity and quality of life. Results of the ALMANAC randomised trial comparing sentinel node biopsy with standard axillary treatment in the management of patients with early breast cancer. Breast Cancer Res Treat. 2006; 95 (3): 279-93.

10. Latosinsky S, Dabbs K, Moffat F; Evidence-Based Reviews in Surgery Group. Canadian Association of General Surgeons and American College of Surgeons Evidence-Based Reviews in Surgery. 27. Quality-of-life outcomes with sentinel node biopsy versus standard axillary treatment in patients with operable breast cancer. Randomized multicenter trial of sentinel node biopsy versus standard axillary treatment in operable breast cancer: the ALMANAC Trial. Can J Surg 2008; 51 (6): 483-5.

11. Gill G, SNAC Trial Group of the Royal Australasian College of Surgeons (RACS) and NHMRC Clinical Trials Centre. Sentinel-lymph-node-based management or routine axillary clearance? One-year outcomes of sentinel node biopsy versus axillary clearance (SNAC): a randomized controlled surgical trial. Ann Surg Oncol 2009; 16 (2): 266-75.

12. Ashikaga T, Krag DN, Land SR, Julian TB, Anderson SJ, Brown AM, et al. Morbidity results from the NSABP B-32 trial comparing sentinel lymph node dissection versus axillary dissection. J Surg Oncol 2010; 102 (2): 111-8.

13. Land SR, Kopec JA, Julian TB, Brown AM, Anderson SJ, Krag DN, et al. Patient-reported outcomes in sentinel node-negative adjuvant breast cancer patients receiving sentinel-node biopsy or axillary dissection: National Surgical Adjuvant Breast and Bowel Project phase III protocol B-32. J Clin Oncol 2010; 28 (25): 3929-36.

14. Mabry H, Giuliano AE. Sentinel node mapping for breast cancer: progress to date and prospects for the future. Surg Oncol Clin N Am 2007; 16 (1): 55-70.

15. Lyman GH, Giuliano AE, Somerfield MR, Benson AB 3rd, Bodurka DC, Burstein HJ, et al. American Society of Clinical Oncology guideline recommendations for sentinel lymph node biopsy in early-stage breast cancer. J Clin Oncol 2005; 23 (30): 7703-20.

16. Krag D, Weaver D, Ashikaga T, Moffat F, Klimberg 
VS, Shriver C, et al. The sentinel node in breast cancera multicenter validation study. N Engl J Med 1998; 339 (14): 941-6.

17. Straver ME, Meijnen $P$, van Tienhoven $G$, van de Velde CJ, Mansel RE, Bogaerts J, et al. Sentinel node identification rate and nodal involvement in the EORTC 10981-22023 AMAROS trial.Ann Surg Oncol 2010; 17 (7): 1854-61.

18. Chen AY, Halpern MT, Schrag NM, Stewart A, Leitch $\mathrm{M}$, Ward E, et al. Disparities and trends in sentinel lymph node biopsy among early-stage breast cancer patients (1998-2005). J Natl Cancer Inst 2008; 100 (7): 462-74.

19. Quan ML, Hodgson N, Lovrics P, Porter G, Poirier B, Wright FC. National adoption of sentinel node biopsy for breast cancer: lessons learned from the Canadian experience. Breast J 2008; 14 (5): 421-7.

20. Heneghan HM, Prichard RS, Devaney A, Sweeney KJ, Malone C, McLaughlin R, et al. Evolution of breast cancer management in Ireland: a decade of change. BMC Surg 2009; 9: 15.

21. Kim T, Giuliano AE, Lyman GH. Lymphatic mapping and sentinel lymph node biopsy in early-stage breast carcinoma: a metaanalysis. Cancer 2006; 106 (1): 4-16.

22. Ravdin PM, De Laurentis M,Vendely T, Lark GM. Prediction of axillary lymph node status in breast cancer patients by use of prognostic indicators. J Natl Cancer Inst 1994; 86 (23): 1771-5.

23. Povoski SP, Olsen JO, Young DC, Clarke J, Burak WE, Walker MJ, et al. Prospective randomized clinical trial comparing intradermal, intraparenchymal, and subareolar injection routes for sentinel lymph node mapping and biopsy in breast cancer. Ann Surg Oncol 2006; 13 (11): 1412-21.

24. Hill AD, Tran KN, Akhurst T, Yeung H, Yeh SD, Rosen $\mathrm{PP}$, et al. Lessons learned from 500 cases of lymphatic mapping for breast cancer. Ann Surg 1999; 229 (4): 52835.

25. Krag DN, Anderson SJ, Julian TB, Brown AM, Harlow SP, Costantino JP, et al. Sentinel-lymph-node resection compared with conventional axillary-lymph-node dissection in clinically node-negative patients with breast cancer: overall survival findings from the NSABP B-32 randomized phase 3 trial. Lancet Oncol 2010; 11 (10): 927-33.

26. Krag D, Weaver D, Ashikaga T, Moffat F, Klimberg VS, Shriver C, et al.The sentinel node in breast cancer-a multicenter validation study. N Engl J Med 1998; 339 (14): 941-6.

27. Giuliano AE, Jones RC, Brennan M, Statman R. Sentinel lymphadenectomy in breast cancer. J Clin Oncol 1997; 15 (6): 2345-50.
28. Veronesi U, Paganelli G, Galimberti V, Viale G, Zurrida $\mathrm{S}$, Bedoni $\mathrm{M}$, et al. Sentinel-node biopsy to avoid axillary dissection in breast cancer with clinically negative lymph-nodes. Lancet 1997; 349 (9069): 1864-7.

29. Albertini JJ, Lyman GH, Cox C, Yeatman T, Balducci $\mathrm{L}, \mathrm{Ku} \mathrm{N}$, et al. Lymphatic mapping and sentinel node biopsy in the patient with breast cancer. JAMA 1996; 276 (22): 1818-22.

30. Borgstein PJ, Pijpers R, Comans EF, van Diest PJ, Boom RP, Meijer S, et al. Sentinel lymph node biopsy in breast cancer: guidelines and pitfalls of lymphoscintigraphy and gamma probe detection. J Am Coll Surg 1998; 186 (3): 275-83.

31. Turner RR, Chu KU, Qi K, Botnick LE, Hansen NM, Glass EC, et al. Pathologic features associated with nonsentinel lymph node metastases in patients with metastatic breast carcinoma in a sentinel lymph node. Cancer 2000; 89 (3): 574-81.

32. Giuliano AE, Kirgan DM, Guenther JM, Morton DL. Lymphatic mapping and sentinel lymphadenectomy for breast cancer. Ann Surg 1994; 220 (3): 391-8; discussion 398-401.

33. Lyman GH, Temin S, Edge SB, Newman LA, Turner RR, Weaver DL, et al. Sentinel lymph node biopsy for patients with early-stage breast cancer: American Society of Clinical Oncology clinical practice guideline update. J Clin Oncol 2014; 32 (13): 1365-83.

34. Olson JA Jr, McCall LM, Beitsch P, Whitworth PW, Reintgen DS, Blumencranz PW, et al. Impact of immediate versus delayed axillary node dissection on surgical outcomes in breast cancer patients with positive sentinel nodes: results from American College of Surgeons Oncology Group Trials Z0010 and Z0011. J Clin Oncol 2008; 26 (21): 3530-5.

35. Andersson Y, Frisell J, Sylvan M, de Boniface J, Bergkvist L. Breast cancer survival in relation to the metastatic tumor burden in axillary lymph nodes. J Clin Oncol 2010; 28 (17): 2868-73.

36. de Boer M, van Dijck JA, Bult P, Borm GF, Tjan-Heijnen VC. Breast cancer prognosis and occult lymph node metastases, isolated tumor cells, and micrometastases. J Natl Cancer Inst 2010; 102 (6): 410-25.

37. Shahar KH, Buchholz TA, Delpassand E, Sahin AA, Ross MI, Ames FC, et al. Lower and central tumor location correlates with lymphoscintigraphy drainage to the internal mammary lymph nodes in breast carcinoma. Cancer 2005; 103 (7): 1323-9.

38. Chen RC, Lin NU, Golshan M, Harris JR, Bellon JR. Internal mammary nodes in breast cancer: diagnosis and implications for patient management-a systematic review.J Clin Oncol 2008; 26 (30): 4981-9. 
39. Mansel RE, Goyal A, Newcombe RG; ALMANAC Trialists Group. Internal mammary node drainage and its role in sentinel lymph node biopsy: the initial ALMANAC experience. Clin Breast Cancer 2004; 5 (4): 279-84.

40. Klauber-DeMore N, Bevilacqua JL, Van Zee KJ, Borgen $\mathrm{P}$, Cody HS 3rd. Comprehensive review of the management of internal mammary lymph node metastases in breast cancer. J Am Coll Surg 2001; 193 (5): 547-55.

41. Tanis PJ, Nieweg OE, Valdés Olmos RA, Peterse JL, Rutgers EJ, Hoefnagel CA et al. Impact of non-axillary sentinel node biopsy on staging and treatment of breast cancer patients. Br J Cancer 2002; 87 (7): 705-10.

42. Heuts EM, van der Ent FW, von Meyenfeldt MF, Voogd AC. Internal mammary lymph drainage and sentinel node biopsy in breast cancer-A study on 1008 patients. Eur J Surg Oncol 2009; 35 (3): 252-7.
43. Tanis PJ, Deurloo EE, Valdés Olmos RA, Rutgers EJ, Nieweg OE, Besnard AP, et al. Single intralesional tracer dose for radio-guided excision of clinically occult breast cancer and sentinel node. Ann Surg Oncol 2001; 8 (10): 850-5.

44. Doting MH, Jansen L, Nieweg OE, Piers DA, Tiebosch AT, Koops HS, et al. Lymphatic mapping with intralesional tracer administration in breast carcinoma patients. Cancer 2000; 88 (11): 2546-52.

45. Chagpar A, Martin RC 3rd, Chao C, Wong SL, Edwards MJ, Tuttle T, et al. Validation of subareolar and periareolar injection techniques for breast sentinel lymph node biopsy. Arch Surg 2004; 139 (6): 614-8.

46. Uren RF, Howman-Giles R, Chung D, Thompson JF. Nuclear medicine aspects of melanoma and breast lymphatic mapping. Semin Oncol 2004; 31 (3): 338-48. 